\title{
Research on Preference Polyhedron Model Based Evolutionary Multiobjective Optimization Method for Multilink Transmission Mechanism Conceptual Design
}

\author{
Haihua Zhu, Jun Yang, Weihua Lu, and Jing Li \\ College of Mechanical and Electrical Engineering, Nanjing University of Aeronautics and Astronautics, Nanjing 210016, China \\ Correspondence should be addressed to Haihua Zhu; zhuhh@nuaa.edu.cn
}

Received 15 October 2015; Revised 4 March 2016; Accepted 31 March 2016

Academic Editor: Thomas Hanne

Copyright (C) 2016 Haihua Zhu et al. This is an open access article distributed under the Creative Commons Attribution License, which permits unrestricted use, distribution, and reproduction in any medium, provided the original work is properly cited.

To make the optimal design of the multilink transmission mechanism applied in mechanical press, the intelligent optimization techniques are explored in this paper. A preference polyhedron model and new domination relationships evaluation methodology are proposed for the purpose of reaching balance among kinematic performance, dynamic performance, and other performances of the multilink transmission mechanism during the conceptual design phase. Based on the traditional evaluation index of single target of multicriteria design optimization, the robust metrics of the mechanism system and preference metrics of decision-maker are taken into consideration in this preference polyhedron model and reflected by geometrical characteristic of the model. At last, two optimized multilink transmission mechanisms are designed based on the proposed preference polyhedron model with different evolutionary algorithms, and the result verifies the validity of the proposed optimization method.

\section{Introduction}

To improve work efficiency of mechanical press and acquire specific kinematic and dynamic output of slider, multilink transmission mechanisms are applied to replace the traditional crank-link mechanisms. The determination of structure parameters is the most important link in the design process of the mechanism, which determines the ultimate characteristics and performances of the product to a great extent, especially in the conceptual design phase. The conceptual development of the product accomplishes almost 70 percent of the performances and characteristics while occupying only 1 percent of the whole life-cycle cost as shown in Figure 1. For this reason, this research focuses on the optimal design problems of the multilink transmission mechanism in the conceptual design phase.

The design optimization of multilink transmission mechanism is competitive not only for kinematic and dynamic performance, but also for manufacturability, serviceability, and overall life-cycle cost. Considering the conflicting objectives, as well as the highly complex search space and constraints, a rigorous and quantitative multidisciplinary design methodology and evaluation standard of design scheme are needed for solving such multiobjective optimization problem (MOOP). The evolutionary algorithms (EAs) could provide efficient solutions to the above problems $[1,2]$. In such optimization problems, the design objectives describe all the product functions as well as the constraints under which these functions should be realized. Instead of a single optimal objective, multitudinous and competing objectives of actual engineering problems often provide a set of compromise solutions, which is generally called Pareto front. As the basis of decision-making, the Pareto front can offer valuable tradeoff information between objectives.

The feasibility of applying evolutionary algorithms to the solutions of multiobjective engineering optimization problems has been explored by many previous researches.

Marler and Arora [3] presented a survey of current continuous nonlinear multiobjective optimization (MDO) concepts and method. It was found that no single method was superior, while the selection of a specific method is depending on the type of information provided in the problem, the user's preference, the solution requirements, and the availability of software. 


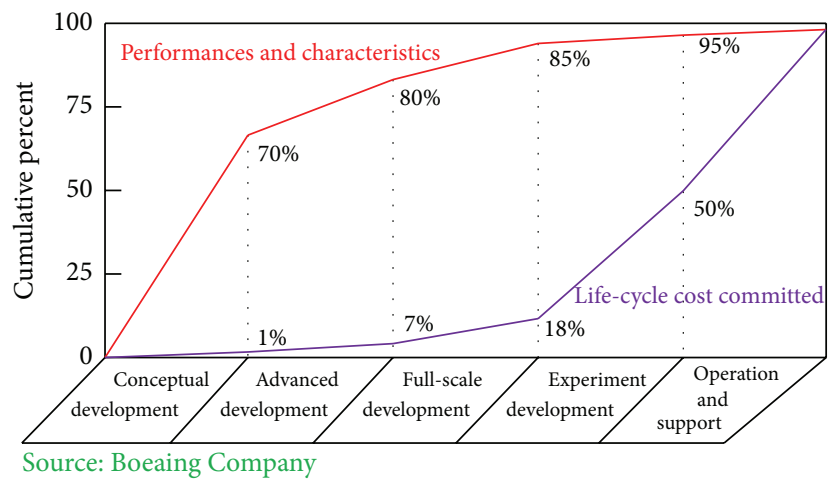

FIGURE 1: Life-cycle cost committed and performances and characteristics of product versus incurred life-cycle phase.

Saravanan et al. [4] realized the multicriteria design optimization of robot grippers by using evolutionary algorithms. Three different robot gripper configurations with five competing objectives, nine constraints, and seven design variables were optimized by using intelligent techniques. What is more, the paper compared the computing time and the quality of the solution obtained from MOGA, NSGA-II, MODE, and the proposed optimization procedures.

Castillo et al. [5] described the use of a genetic algorithm (GA) for the problem of offline point-to-point autonomous mobile robot path planning based on two criteria, length and difficulty, and showed that GAs were effective tools for solving such path-planning problems.

However, the researches mentioned above are based on traditional ranking and domination method. This research aims to extend the evaluation process of Pareto front solutions with the incorporation of robust design and concept of multiobjective risk decision. A preference polyhedron model is proposed to provide further dominant relationship information [6]. Thus, the Pareto front can be further improved and more valuable optimization results can be obtained. The rest of this paper consists of the following-Section 2 reviews some literatures. Section 3 introduces the proposed methodology and the key technologies for multilink transmission mechanism design. Section 4 describes the establishment of optimization model. And the experimental results are depicted in Section 5. Finally, the conclusions and further work are summarized in Section 6.

\section{Basic Theory}

This section will introduce the main concepts and previous research works related to this project, covering topics like basic evolutionary algorithm theory, multiobjective optimization, existing Pareto optimal front, and design optimization of multilink transmission mechanism. Moreover, the basic conception of preference polyhedron model will also be introduced briefly in this section.

2.1. Evolutionary Algorithms. Evolutionary algorithms are random exploring optimization algorithm based on the idea of the biological evolutionary. Different from traditional optimization algorithms, evolutionary algorithms have no strict requirements for the problem. After a repetitive loop or a series of generations, it can find the fittest individual or individuals to solve the particular problem. Thus, evolutionary algorithms are widely used to solve complex optimization problems $[7,8]$.

Genetic algorithm is a typical example of evolutionary algorithms, which regards each solution to the particular optimization problem as an individual in the evolution process of large population. The fitness of each individual is determined by a given fitness function which evaluates the level of aptitude that particular individual solves the given optimization problem, while each generation in the evolution process will create a new set of individuals through genetic operators: crossover and mutation operation. New child individuals produced by the above operations will then be selected by a selection method and finally reinserted into the population by a replacement method. Such process will be repeated generationally at a user defined number. While solving real engineering design problems, for example, to observe the optimal structure parameters of a complex mechanical system, the individual characteristics always can be coded into a finite set of such design parameters. These parameters make up the chromosome with genetic information that represents the real-world structure of the individual, which in this case is a solution to the particular optimization problem. The process can be concluded as shown in Figure 2.

2.2. Multiobjective Optimization Problems. In a real complex engineering optimization problem, lots of various performance factors or characteristic factors should be taken into consideration while such factors are always difficult to be quantified and competing with each other [9-11]. As a result, in such multiobjective optimization problems, it is difficult to reach an optimal solution with respect to all of the objectives evaluated individually. Creating a single-objective function or converting the objectives into restrictions imposed on the optimization problem is one of the conventional ways [12-15]. For example, the evaluation function of a specific problem can be expressed as minimizing or maximizing the fitness evaluation function $F(i)$ :

$$
F(i)=\sum_{i=1}^{L} \omega_{i} f_{i},
$$




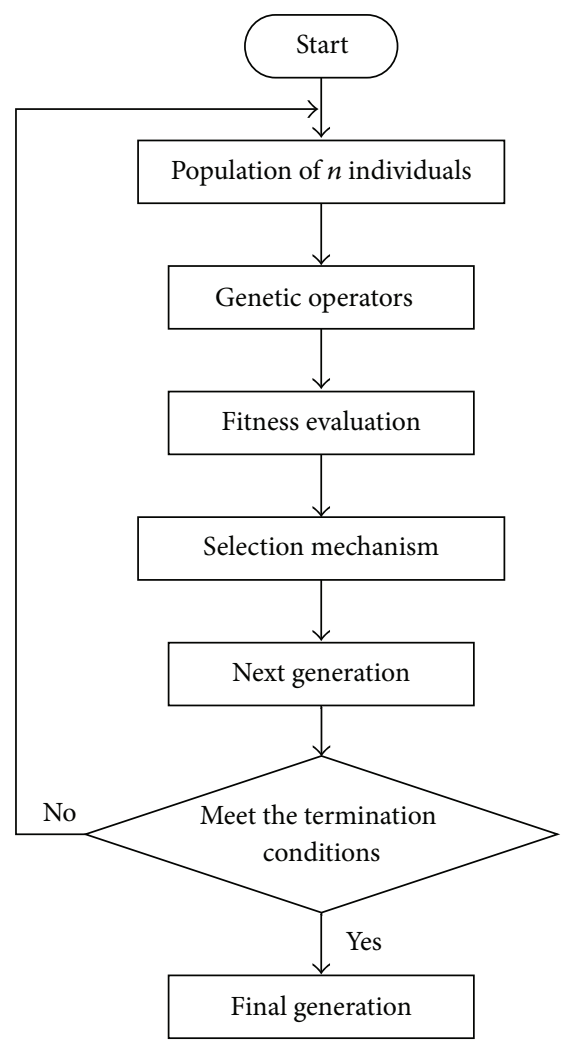

FIGURE 2: Standard process of evolutionary algorithms.

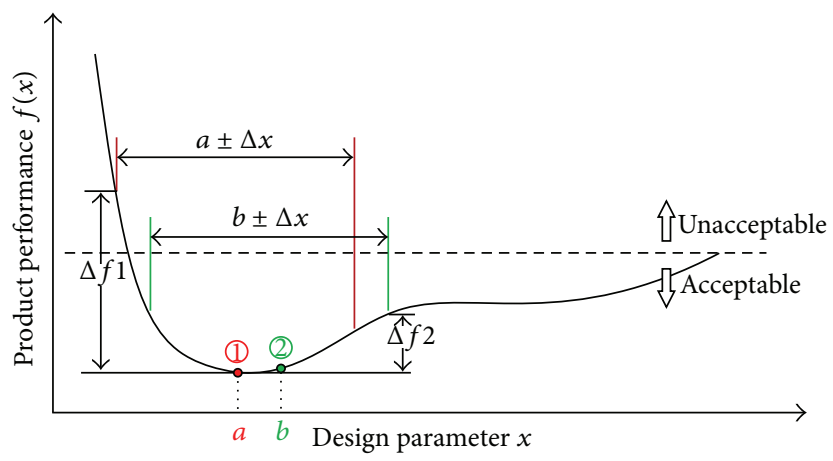

(1) Deterministic optimal design point
(2) Robust optimal design point
$\Delta x$ : error due to uncertainty factors

FIGURE 3: Deterministic optimal design point and robust optimal design point.

where $\omega_{i}$ is weight factor, reflecting the contribution of each subobjective to the final evaluation function, and $f_{i}$ is the value of $i$ th subobjective.

However, the above method always overlooks the stability of the optimization result in the optimum iterative procedure. Figure 3 shows a typical example about deterministic optimal design point and robust optimal design point. As a result of the existence of error due to uncertainty factors (such as manufacturing and fixing error, external disturbance, and fatigue wear), the real product performances should be considered as an interval. Although the deterministic optimal design point provides the best performance, the performance fluctuation $\Delta f$ of the deterministic optimal design point may be larger than the other design points when the product performance is considered within the same and specific interval range. Sometimes the performance fluctuation due to error caused by uncertainty factors may be beyond the limit of design objective, so it is necessary to sacrifice some performances to get a more reliable and steady designing scheme.

2.3. Preference Polyhedron Model. Considering the interaction of different objectives in the multiobjective optimization 


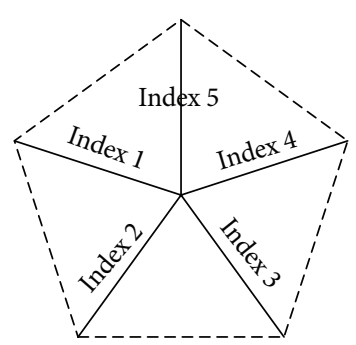

Model 1

$$
F(1)=\sum_{i=1}^{L} \omega_{i} f_{i}(X)
$$

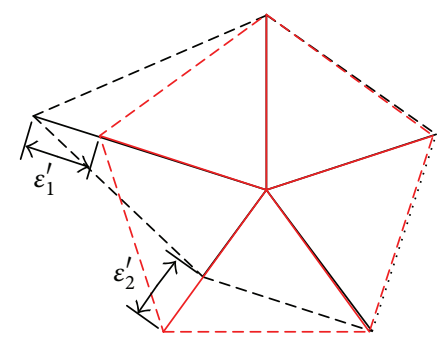

Model 2

$$
F(2)=\sum_{i=1}^{L} \omega_{i} f_{i}^{\prime}(X)
$$

FIGURE 4: Preference polyhedron model.

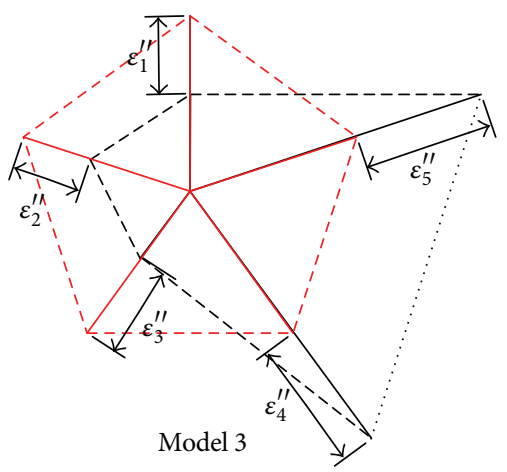

$$
F(3)=\sum_{i=1}^{L} \omega_{i} f_{i}^{\prime \prime}(X)
$$

problems, it is necessary to evaluate the importance of multiple objectives and determine the degree to which objectives need to be modified [16-18]. Human elements must be regarded as a key component in any successful system design methodology. As mentioned above, many existing methods for solving such multiobjective optimization problems were developed, and the human elements always took part in the iterative optimization process based on the concept of fuzzy mathematics and theory of probability $[19,20]$. To better evaluate the optimal Pareto front, extended evaluation standards used to make further reflection to the quality of the alternative have been defined, for example, graphical illustration. In order to select the most preferred alternative, Miettinen researched different graphical visualization tools used to support and help the decision-maker in understanding similarities and differences between the alternatives and graphical illustration [21]. Moreover, Trinkaus and Hanne represented a novel multicriteria decision support system (MCDSS) to solve multicriteria decision problems [22]. Visualization methods are both used in the above two researches for better description and analysis of the alternatives. In this paper, a preference polyhedron model is proposed and used to describe the alternatives, as shown in Figure 4. Under the basis of the description of the alternatives, the alternatives could be filtered by using the proposed preference polyhedron model. And the most preferred alternative satisfying the preference of decision-makers will be automatically obtained.

The geometric size and shape parameters of the polygon models in Figure 4 reflect different evaluation information including quantized value of different design index and the stability of whole performance. For example, the length parameter of the full line in Figure 4 can reflect the value of the $i$ th indicator $f(i)$, and then it is easy to get the global evaluation function $F$. However, the evaluation function of a specific problem should not include only evaluation function $F$; the error function reflecting the stability of performance should also be taken into consideration and can be defined as follows:

$$
E(i)=\sum_{i=1}^{L} \omega_{i} \varepsilon_{i} .
$$

Moreover, the above polygon model can offer more geometric size and shape information which can be used to build extra evaluation function to improve the Pareto front of optimal results when the evaluation function $F(1)=F(2)=$ $F(3)$. In contrast with the proposed methods in [23-25] all centered around the concept of Pareto optimality and the Pareto optimal set, in this paper, the preference of decisionmaker and the risk assessment of design scheme based on the area attribute of polygon model are used to define related dominant relationship.

\subsection{Design Optimization Problem of Multilink Transmission} Mechanism. The design optimization problem of multilink transmission mechanism is a typical multiobjective optimization problem, where various factors such as the kinematic performance, the dynamic performance, the life-cycle cost, manufacturability, and serviceability should be considered. This means that the evolutionary should optimize possible structure parameters based on at least such following criteria: kinematic performance, dynamic performance, manufacturing costs, and structural stability. In addition, the design parameters of multilink transmission mechanism include the length of each link and the angle parameters of component placement, which will ultimately influence the performance of the mechanism. The design parameters are usually defined as a design vector $X$ which contains all variables that form the design space. The design vector of this transmission mechanism can be expressed as follows:

$$
X=\left[\begin{array}{c}
x_{1} \\
x_{2} \\
x_{3} \\
x_{i} \\
\vdots \\
x_{n}
\end{array}\right]=\left[\begin{array}{c}
\text { steric configuration }[-] \\
\text { link-length parameters }[\mathrm{mm}] \\
\text { material parameters }[-] \\
\text { layout angle }[\text { degree }] \\
\vdots \\
\text { output torque }[\mathrm{N} \cdot \mathrm{M}]
\end{array}\right] \text {. }
$$

The objective of the design of multilink transmission mechanism can be represented as a vector $J$. It can be divided 


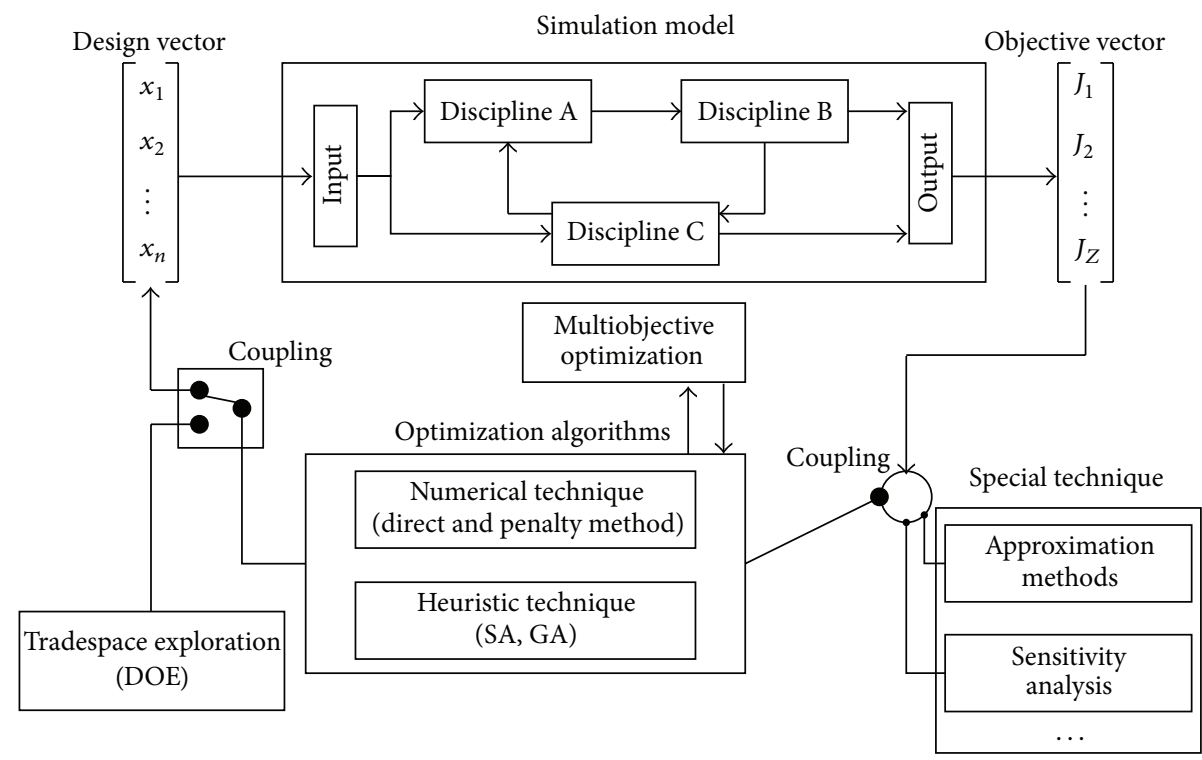

FIGURE 5: Multiobjective optimization design (MOOD) framework.

into $z$ different types, and each of them includes several subobjectives:

$$
J=\left[\begin{array}{c}
J_{1} \\
J_{2} \\
J_{3} \\
J_{i} \\
\vdots \\
J_{z}
\end{array}\right]=\left[\begin{array}{c}
\text { cost }[\$] \\
\text { range }[\mathrm{mm}] \\
\text { weight }[\mathrm{kg}] \\
\text { nominal pressure }[\mathrm{kn}] \\
\vdots \\
\text { mechanical efficiency }[\%]
\end{array}\right] .
$$

It is the most important thing to determine an optimal parameter set $X^{\prime}$ which can make the objective vector the balance of state where all disciplines can complement each other. However, interactions and conflictions among the above elements increase the difficulty of optimal solution of this complex engineering problem. The multiobjective optimization algorithm offered an effective solution for such problem. The typical framework of MOOD has been shown in Figure 5.

\section{Optimization Model for Multilink Transmission Mechanism Design}

This section briefly introduces the input and output parameters in the conceptual design process of multilink transmission mechanism. Moreover, the preference polyhedron model and related dominant relationship will also be introduced in detail in this section. By the iteration of the proposed optimization model, new multiobjective optimal solution based on risk assessment and robust design will ultimately be adopted. Figure 6 shows a comparison between the traditional crank-link mechanism and multilink transmission mechanism, which are both driven by a motor on the point $O$ and ultimately turn the rotational motion of motor to the straight reciprocating motion of the slider by the connecting rods.

3.1. Design Parameters and Constraint Equations. This simplified model of multilink transmission mechanism was derived from a real multilink mechanical press, so the design variables depend upon the original value of real design parameters. The design vector $X$ and objective vector $J$ can be expressed as follows:

$$
X=\left[\begin{array}{c}
L_{\mathrm{OA}} \\
L_{\mathrm{AB}} \\
L_{\mathrm{BC}} \\
L_{\mathrm{OC}} \\
L_{\mathrm{CD}} \\
L_{\mathrm{DE}} \\
\alpha
\end{array}\right]
$$

$$
=\left[\begin{array}{c}
\text { the length of link OA }[\mathrm{mm}] \\
\text { the length of link } \mathrm{AB}[\mathrm{mm}] \\
\text { the length of link BC }[\mathrm{mm}] \\
\text { the length of link OC }[\mathrm{mm}] \\
\text { the length of link CD }[\mathrm{mm}] \\
\text { the length of link DE }[\mathrm{mm}] \\
\text { the angle between link BC and CD }[\text { degree }]
\end{array}\right],
$$



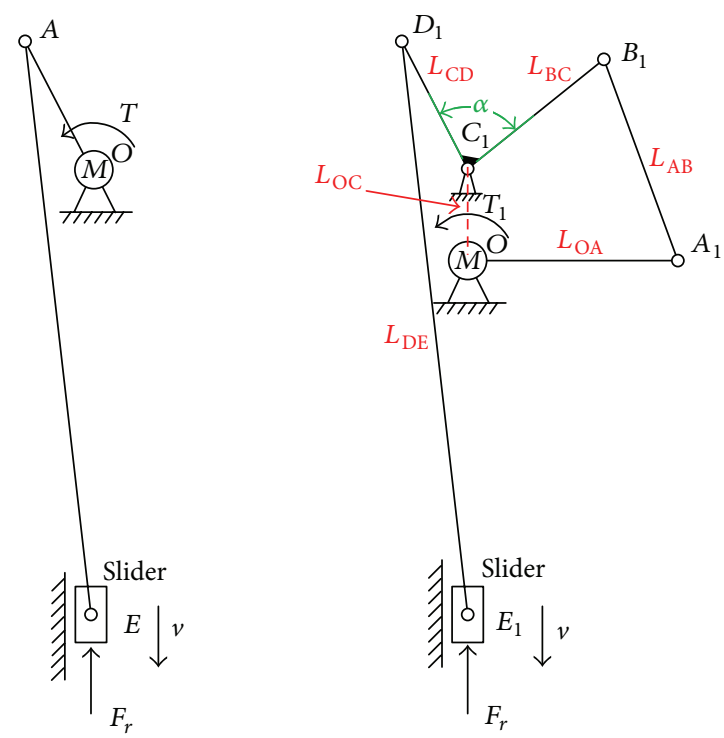

FIGURE 6: The traditional crank-link and multilink transmission mechanism.

$$
\begin{aligned}
J & =\left[\begin{array}{l}
T \\
v \\
k \\
a \\
y
\end{array}\right] \\
& =\left[\begin{array}{c}
\text { input torque }[\mathrm{N} \cdot \mathrm{mm}] \\
\text { the velocity of the slider }[\mathrm{mm} / \mathrm{s}] \\
\text { stoke speed ratio }[-]
\end{array}\right] .
\end{aligned}
$$

The basic kinematic and dynamic performances and other performances concerned in this optimization are all defined by the elements of the vector $J$ and closely associated with the value of the design parameter in vector $X$. In order to meet some geometric dimensions and motion requirements, some constraint equations must be satisfied during the optimization process. In addition, some elements in objective vector $J$ should be transformed into constraints to simplify the optimization model. These constraint equations are as follows:

$$
\begin{aligned}
& \max \left(L_{\mathrm{OA}}, L_{\mathrm{AB}}, L_{\mathrm{BC}}, L_{\mathrm{OC}}\right) \\
& \quad+\min \left(L_{\mathrm{OA}}, L_{\mathrm{AB}}, L_{\mathrm{BC}}, L_{\mathrm{OC}}\right) \\
& \leq \frac{L_{\mathrm{OA}}+L_{\mathrm{AB}}+L_{\mathrm{BC}}+L_{\mathrm{OC}}}{2}, \\
& v \in\left[v_{r}-\Delta v, v_{r}+\Delta v\right], \\
& k \in\left[k_{r}-\Delta k, k_{r}+\Delta k\right], \\
& a \in\left[a_{r}-\Delta a, a_{r}+\Delta a\right],
\end{aligned}
$$

where $v_{r}, k_{r}, a_{r}$ are ideal design index and $\Delta v, \Delta k, \Delta a$ are reasonable deviation.

So, the optimization model is simplified to determine the optimal set of vectors $X$ to maximize or minimize the objective $J$ under the above constraints, where the vector $J$ is simplified as follows:

$$
J=\left[\begin{array}{l}
T \\
y
\end{array}\right]=\left[\begin{array}{c}
\text { input torque }[\mathrm{N} \cdot \mathrm{mm}] \\
\text { the cost of the mechanim }[\$]
\end{array}\right] .
$$

As a result of the error of the manufacturing and assembling, the design parameters always deviate from the ideal optimal design value and bring about the fluctuation of the output performance. The evaluation function $E$ of such performance fluctuation is defined in the following way:

$$
E(i)=\sum_{i=1}^{L} \omega_{i} \varepsilon_{i}
$$

where in (9) $\varepsilon_{i}$ reflects the performance fluctuation and $\omega_{i}$ reflects the impact of such fluctuation on the whole evaluation.

3.2. Preference Polyhedron Model and Related Dominant Relationship. The above multiobjective optimization problems were ultimately expressed by the design vector $X$ expressed by (5), the objective vector $J$ expressed by (6), the evaluation function $E$ expressed by (9), and some constraints. And multiple objectives are combined into scalar objective via weight vector, which can be determined randomly or adaptively through direct assignment, eigenvector method, empty method, and minimal information method. However, if the objective function is simply weighted and added to produce a single fitness, the value of the ultimate evaluation function may be dominated by the objective with large range and small weight. To avoid such condition, the first step to establish 
the optimization model is to normalize each objective. The normalizing condition can be defined in the following way:

$$
f_{i}(X)=\frac{f_{i}^{\prime}(X)}{\min _{X \in D} f_{i}^{\prime}(X)},
$$

where in (10) $D$ is the feasible domain of design vector $X$ and $\min _{X \in D} f_{i}^{\prime}(X)$ is the lower limit of the value of objective function. In this way, all objective functions can be evaluated in the same metric. The preference polyhedron model can be created as shown in Figure 7.

The practical engineering problems always have many upper and lower limits, and the inside and outside red lines of the polyhedron in Figure 7 reflect such limits. The length of radial lines sent out from the center of the polyhedron reflects the value of object function $f_{i}(X)$. The parameter $\varphi_{i}$ is determined by the angle between adjacent objective functions. Based on the definition of the confidence interval, the overall and individual confidence interval is expressed by the area of polyhedrons $S$ and $s$. The confidence level $P$ of each individual in the iterative optimization process can be defined as follows:

$$
P=\sum_{i=1}^{L} \varphi_{i} r_{i}
$$

Equation (11) can be described by the following:

$$
\begin{aligned}
P & =\sum_{i=1}^{L} \varphi_{i} \frac{(1 / 2) f_{i}(X) \cdot \sin \left(\varphi_{i} \cdot 2 \pi\right) \cdot f_{i+1}(X)-(1 / 2) f_{i}(X)_{\min } \cdot \sin \left(\varphi_{i} \cdot 2 \pi\right) \cdot f_{i+1}(X)_{\min }}{(1 / 2) f_{i}(X)_{\max } \cdot \sin \left(\varphi_{i} \cdot 2 \pi\right) \cdot f_{i+1}(X)_{\max }-(1 / 2) f_{i}(X)_{\min } \cdot \sin \left(\varphi_{i} \cdot 2 \pi\right) \cdot f_{i+1}(X)_{\min }} \\
& =\sum_{i=1}^{L} \varphi_{i} \frac{f_{i}(X) f_{i+1}(X)-f_{i}(X)_{\min } f_{i+1}(X)_{\min }}{f_{i}(X)_{\max } f_{i+1}(X)_{\max }-f_{i}(X)_{\min } f_{i+1}(X)_{\min }},
\end{aligned}
$$

where the above equation should meet the following constraint equations:

$$
\begin{gathered}
\sum_{i=1}^{L} \varphi_{i}=1, \\
f_{i}(X)_{\min }<f_{i}(X)<f_{i}(X)_{\max } .
\end{gathered}
$$

When the value of $f_{i}(X)$ is out of limit just as shown in Figure 7 , a penalty function is brought into the confidence function to reduce or increase the confidence level of the individual. The penalty functions are divided into two kinds of situations.

Definition 1. As shown in Figure 8, when $f_{i}(X)_{\max }<f_{i}(X)$ and $f_{i+1}(X)<f_{i+1}(X)_{\max }$, the penalty function is defined as follows:

$$
\begin{aligned}
P_{i} & =f\left(f_{i}(X), f_{i+1}(X), f_{i}(X)_{\max }, f_{i+1}(X)_{\max }, \varphi_{i}\right) \\
& =p_{i} \cdot S_{+} \\
& =p_{i} \frac{1}{2} \cdot \frac{\sin \alpha \cdot \sin \beta \cdot\left(f_{i}(X)-f_{i}(X)_{\max }\right)^{2}}{\sin (\alpha+\beta)} .
\end{aligned}
$$

In this definition, $p_{i}$ is penalty factor which is related to the importance level of the subobjective, the parameter $\alpha$ is the angle between $f_{i}(X)$ and $f_{i+1}(X)$ and the parameter $\beta$ is the angle between $f_{i}(X)_{\max }$ and $f_{i+1}(X)_{\max }$. What is more, based on the geometric relationship, the above parameters meet the following constraint equations:

$$
\begin{aligned}
\frac{f_{i+1}(X)}{\sin \alpha} & =\frac{f_{i}(X)}{-\sin \left(\varphi_{i} \cdot 2 \pi+\alpha\right)}, \\
\frac{f_{i+1}(X)}{\sin \alpha} & =\frac{f_{i}(X)}{-\sin \left(\varphi_{i} \cdot 2 \pi+\alpha\right)} .
\end{aligned}
$$

Definition 2. When $f_{i}(X)<f_{i}(X)_{\min }$ and $f_{i+1}(X)_{\min }<$ $f_{i+1}(X)$, the penalty function is defined as follows:

$$
\begin{aligned}
& P_{i}=f\left(f_{i}(X), f_{i+1}(X), f_{i}(X)_{\min }, f_{i+1}(X)_{\min }, \varphi_{i}\right) \\
& \quad=p_{i} \cdot S_{-} \\
& \quad=p_{i} \frac{1}{2} \cdot \frac{\sin \alpha \cdot \sin \beta \cdot\left(f_{i}(X)_{\min }-f_{i}(X)\right)^{2}}{\sin (\alpha+\beta)}, \\
& \frac{f_{i+1}(X)}{\sin \alpha}=\frac{f_{i}(X)}{-\sin \left(\varphi_{i} \cdot 2 \pi+\alpha\right)}, \\
& \frac{f_{i+1}(X)_{\min }}{\sin \beta}=\frac{f_{i}(X)_{\min }}{\sin \left(\beta-\varphi_{i} \cdot 2 \pi\right)} .
\end{aligned}
$$

The confidence function can be ultimately revised as the following form:

$$
\begin{aligned}
P & =\sum_{i=1}^{L} \varphi_{i} \frac{f_{i}(X) f_{i+1}(X)-f_{i}(X)_{\min } f_{i+1}(X)_{\min }}{f_{i}(X)_{\max } f_{i+1}(X)_{\max }-f_{i}(X)_{\min } f_{i+1}(X)_{\min }} \\
& +p_{i} \cdot S_{ \pm}
\end{aligned}
$$

In this way, the individual with high confidence level will be easier to be adopted to compare with the lower ones. Based on the definitions above, two individuals in the Pareto front (as shown in Figure 9) can be further compared by the confidence level in the following ways.

It is assumed that $f_{i}\left(x_{1}, c_{i}\right)$ and $f_{i}\left(x_{2}, c_{i}\right)$, respectively, indicate the value of the $i$ th subobjective for the design vector $x_{1}, x_{2}$, while $f_{i+1}\left(x_{1}, c_{i+1}\right)$ and $f_{i+1}\left(x_{2}, c_{i+1}\right)$ indicate the $(i+1)$ th subobjective. Based on the definition above, the domination relationship can be evaluated by the confidence 


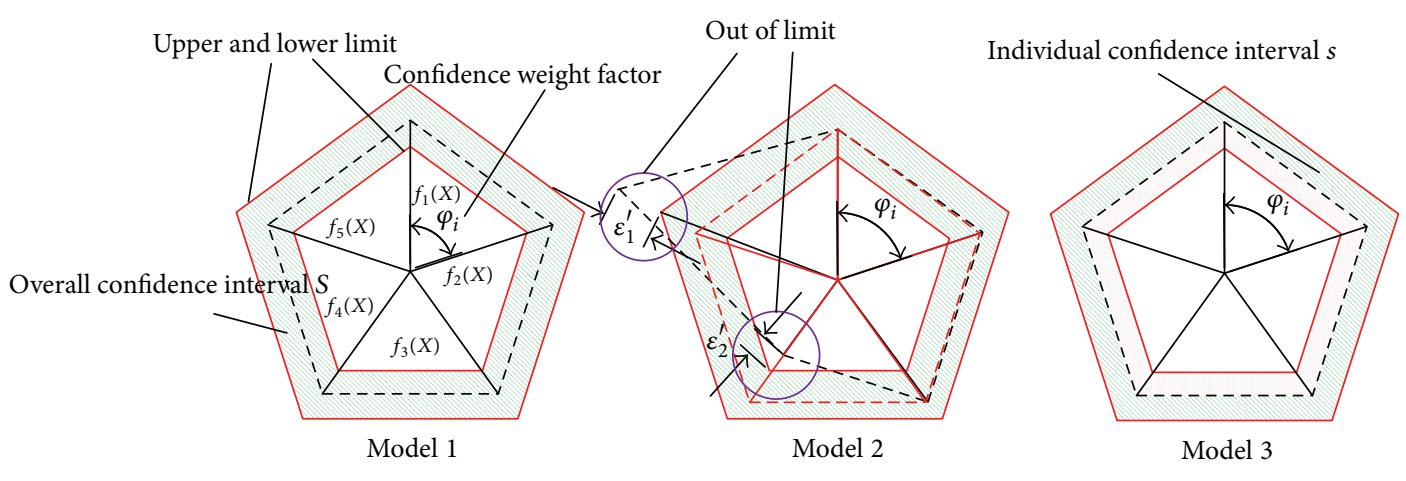

Figure 7: Preference polyhedron model.
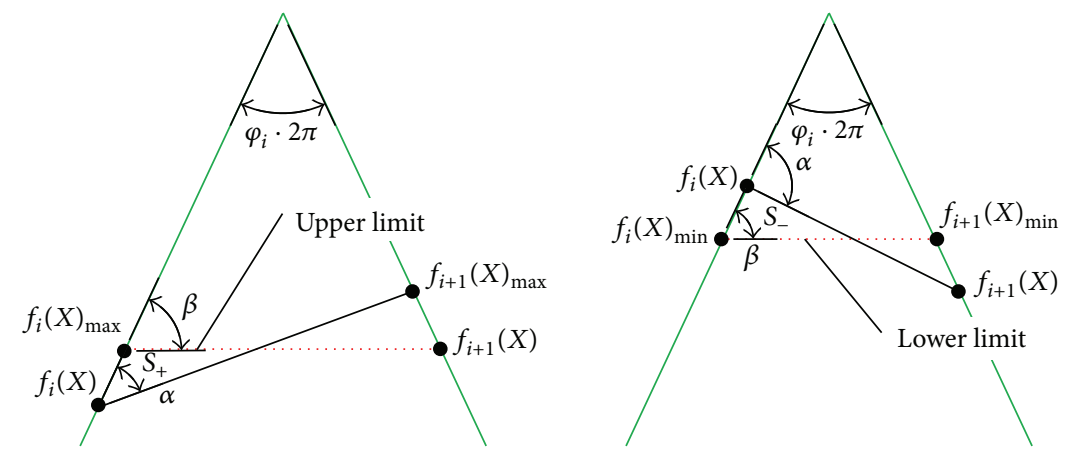

Figure 8: Out of limit.

level (the reliability of $x_{1}$ dominating $x_{2}$ ) and defined as follows:

$$
\sigma\left(x_{1}, x_{2}, c_{i}\right)= \begin{cases}0, & S_{1} \cap S_{2}=S_{2} \\ 1, & S_{1} \cap S_{2}=S_{1},\end{cases}
$$

while in (18), if $S_{1} \cap S_{2} \neq S_{1}$ and $S_{1} \cap S_{2} \neq S_{2}$, (18) can be corrected as follows:

$$
\sigma\left(x_{1}, x_{2}, c_{i}\right)=\frac{S_{1}-S_{1} \cap S_{2}}{S_{1} \cup S_{2}-S_{1} \cap S_{2}} .
$$

Apparently, it can be easily proved that $\sigma\left(x_{1}, x_{2}, c_{i}\right)+$ $\sigma\left(x_{2}, x_{1}, c_{i}\right)=1$, so that the fact that $x_{1}$ dominate $x_{2}$ when $\sigma\left(x_{1}, x_{2}, c_{i}\right) \geq 0.5$ can be true. However, it is necessary to increase the value of the right-hand side of the inequality function to decrease the risk of decision-making.

\section{The Establishment of Optimization Model}

Different metal materials show different malleability under different working conditions including working speed and working temperature. In order to obtain high forming quality products with less energy consumption, the end effector of the transmission mechanism must satisfy some specific kinematic and dynamic performance requirements. More specifically, the slider of press should maintain constant working velocity and acceleration during the working process. In addition, the transmission mechanism should also have a good effect on the reinforcement to consume less energy. Based on the original structure design parameters
TABle 1: Main design objectives.

\begin{tabular}{lcc}
\hline $\begin{array}{l}\text { Design } \\
\text { objectives }\end{array}$ & Description & Value \\
\hline$F_{r}$ & Nominal press $(\mathrm{KN})$ & 2500 \\
$n$ & Slider stroke per minute (times/minute) & 10 \\
$h$ & Slider stroke $(\mathrm{mm})$ & $350 \sim 500$ \\
$h_{n}$ & Nominal stroke $(\mathrm{mm})$ & 8 \\
$k$ & Stroke speed radio & $\geq 1.4$ \\
$v_{i}$ & Ideal working speed of slider $(\mathrm{mm} / \mathrm{s})$ & 70 \\
\hline
\end{tabular}

TABLE 2: Initial structural design parameters for multilink mechanism.

\begin{tabular}{lcccccc}
\hline$L_{\mathrm{OA}}$ & $L_{\mathrm{AB}}$ & $L_{\mathrm{BC}}$ & $L_{\mathrm{OC}}$ & $L_{\mathrm{CD}}$ & $L_{\mathrm{DE}}$ & $\alpha$ \\
\hline $490 \mathrm{~mm}$ & $420 \mathrm{~mm}$ & $360 \mathrm{~mm}$ & $115 \mathrm{~mm}$ & $190 \mathrm{~mm}$ & $1160 \mathrm{~mm}$ & $45^{\circ}$
\end{tabular}

and performance parameters, this section will discuss the establishment of the multiobjective optimization model.

4.1. The Initial Design Information. The main design objectives in this research are demonstrated in Table 1.

The initial values of input and output parameters for the multilink transmission mechanisms in Figure 6 are proposed in Tables 2 and 3. The initial values of structural design parameters are demonstrated in Table 2. Under the premise of meeting the basic design objectives, the real corresponding output performance parameters are demonstrated in Table 3. 

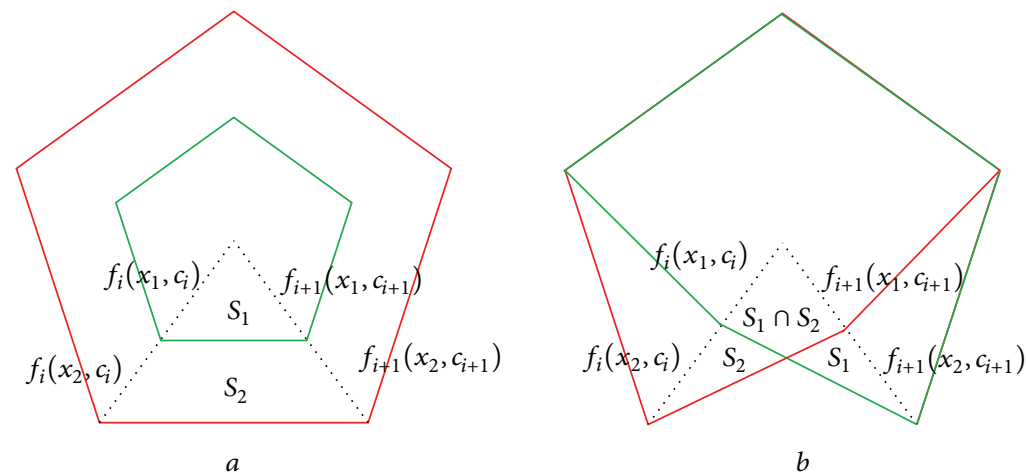

Figure 9: Individuals in Pareto front.

TABLE 3: Output performance parameters.

\begin{tabular}{lcc}
\hline Output parameter & Description & Value \\
\hline$T_{p}(\mathrm{~N} \cdot \mathrm{mm})$ & Peak torque of the motion $(\mathrm{N} \cdot \mathrm{mm})$ & $1.93 \times 10^{8}$ \\
$\bar{T}(\mathrm{~N} \cdot \mathrm{mm})$ & Average torque of the motion $(\mathrm{N} \cdot \mathrm{mm})$ & $8.16 \times 10^{7}$ \\
$k$ & Stroke speed radio & 1.45 \\
$\bar{v}(\mathrm{~mm} / \mathrm{s})$ & Average working velocity of slider $(\mathrm{mm} / \mathrm{s})$ & 125.20 \\
$e_{v}(\mathrm{~mm} / \mathrm{s})$ & Maximum deviation of working speed & 36.19 \\
$\bar{a}\left(\mathrm{~mm}^{2} / \mathrm{s}\right)$ & Average working acceleration of slider $\left(\mathrm{mm}^{2} / \mathrm{s}\right)$ & 40.47 \\
\hline
\end{tabular}

TABLE 4: The preference of design parameters.

\begin{tabular}{lccc}
\hline Parameter & Lower & Value & \\
& Base & Upper \\
\hline$L_{\mathrm{OA}}(\mathrm{mm})$ & 450 & 490 & 550 \\
$L_{\mathrm{AB}}(\mathrm{mm})$ & 350 & 420 & 450 \\
$L_{\mathrm{BC}}(\mathrm{mm})$ & 330 & 360 & 390 \\
$L_{\mathrm{OC}}(\mathrm{mm})$ & 100 & 115 & 130 \\
$L_{\mathrm{CD}}(\mathrm{mm})$ & 175 & 190 & 250 \\
$L_{\mathrm{DE}}(\mathrm{mm})$ & 1000 & 1160 & 1500 \\
$\alpha\left(^{\circ}\right)$ & 40 & 45 & 50 \\
\hline
\end{tabular}

TABLE 5: Output performance variation.

\begin{tabular}{lcc}
\hline Performance & \multicolumn{2}{c}{ Value } \\
& Min & Max \\
\hline $\bar{v}(\mathrm{~mm} / \mathrm{s})$ & 122.47 & 139.98 \\
$\bar{a}\left(\mathrm{~mm}^{2} / \mathrm{s}\right)$ & 35.55 & 43.68 \\
$\bar{T}(\mathrm{~N} \cdot \mathrm{mm})$ & $1.01 \times 10^{8}$ & $1.21 \times 10^{8}$ \\
\hline
\end{tabular}

4.2. The Design Information of Optimization Model. In order to confirm the value range of design parameters, correlation analysis is implemented between input and output parameters. Figure 10 is the correlation graph, which conveys the sensitivity information of output performance parameters to different design parameters.

From Figure 10, where the blue bars mean that the design variables are positively related to the output performance while the red bars indicate the negative correlations, it is obvious that the design parameter $L_{\mathrm{CD}}$ is the most sensitive design parameter for the output parameters $\bar{T}, \bar{v}$, and $\bar{a}$, which should be set at a large range of value under specific constraints. Thus, the preference of design parameters is determined as shown in Table 4.

4.3. The Information of Performance Stability. It is supposed that the error of design parameters in the processing and installation is $\pm 5 \mathrm{~mm}$ and $\pm 2^{\circ}$ and follows the standard distribution. The effect caused by such errors is shown in Table 5 by random combination tests.

4.4. The Optimization Flow of the Model. The basic process of this optimization is just as shown in Figure 11; the most outstanding characteristic is the selection mechanism in contrast with the traditional optimization process which is based on the genetic algorithm. In the selection mechanism, individuals based on the multiobjectives weighted value created by the genetic operators will be ranked firstly. And, then, the first $2 n$ individuals will be selected to go into the next phase. After that, the above $2 n$ individuals will be ranked based on the level of confidence once again and finally the first $n$ individuals will be selected to be the ultimate generation.

The optimization process based on the evolutionary algorithms can be deterministic as you want by either changing the number of iterations or other termination criteria. This experiment adopts NSGA-II multiobjective algorithms as the basic framework and takes the number of iterations as the terminal condition. Relative evolutionary parameters are set as Table 6 indicated.

It must be said that the initial values of output parameters $\left(\bar{v}, \bar{a}, \bar{T}, e_{v}\right)$ are taken as the base value. And new evaluation parameters $\left(\bar{v}^{\prime}, \bar{a}^{\prime}, \bar{T}^{\prime}, e_{v}^{\prime}\right)$ are obtained by the iteration where the comparison of dominant relationships among individuals 

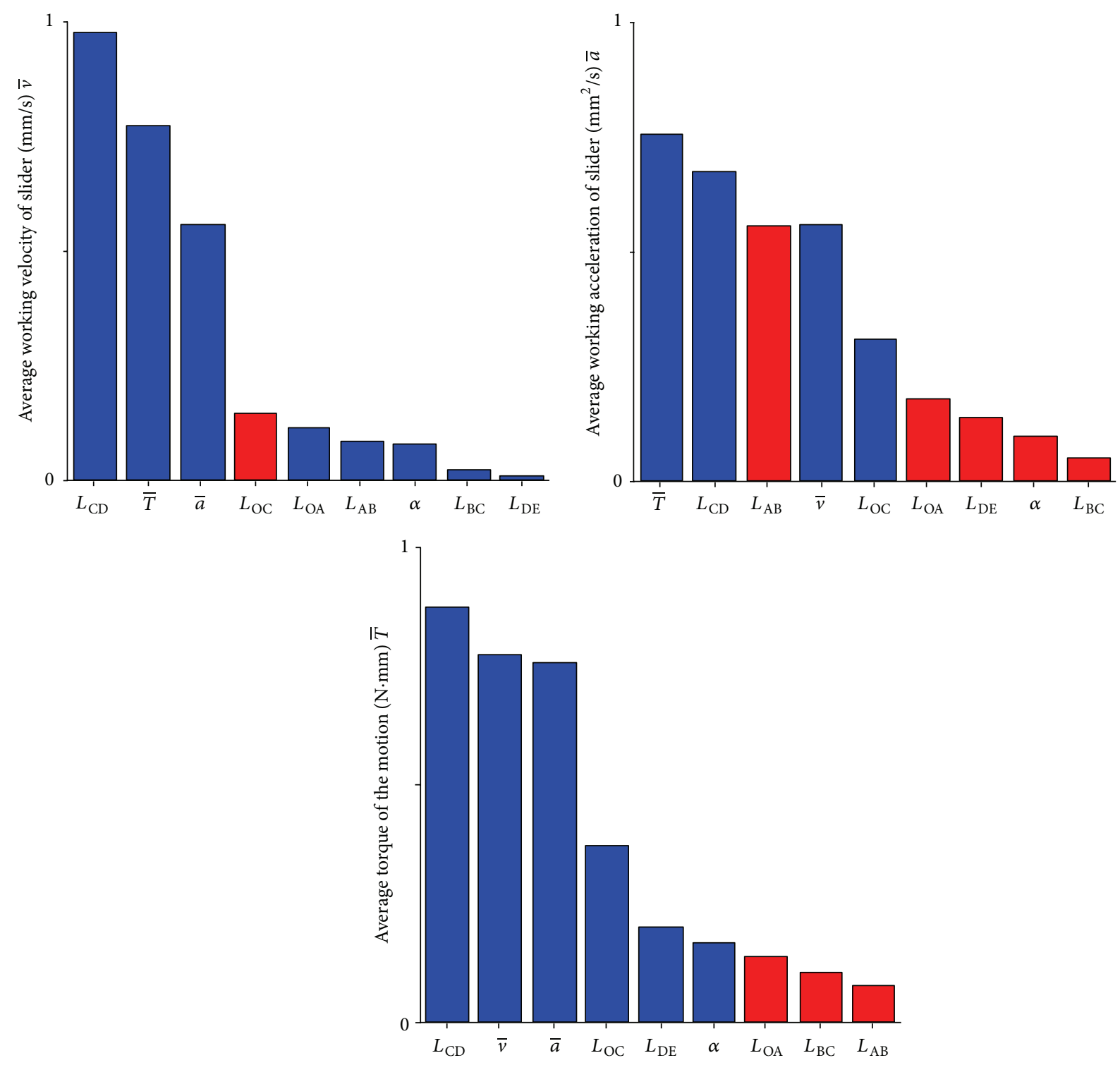

Figure 10: Correlation graph.

TABLE 6: NSGA-II evolutionary parameters.

\begin{tabular}{lc}
\hline Option & Value \\
\hline Population size & 40 \\
Numbers of generations & 200 \\
Crossover probability & 0.8 \\
Crossover distribution index & 10 \\
Mutation distribution index & 20 \\
\hline
\end{tabular}

is implemented by the preference polyhedron model based on (11) to (17) in Section 3.2.

\section{Experimental Results}

The information about new evaluation parameters $\left(\bar{v}^{\prime}, \bar{a}^{\prime}, \bar{T}^{\prime}, e_{v}^{\prime}\right)$ can be obtained, after rationalizing and quantifying the product design problem by selecting appropriate objective functions, design variables, parameters, and constraints. Figures 12, 13, 14, and 15 indicate the history data of the parameters $\bar{v}^{\prime}, \bar{a}^{\prime}, \bar{T}^{\prime}$, and $e_{v}^{\prime}$ separately. From these figures, it can be shown that, with the increase of the number of iterations, each history data of parameters presents convergent tendency. What is more, the convergent tendency of Figures 12, 14, and 15 is more apparent than that of Figure 13. The reason for this is because the parameter $\bar{a}$ is more sensitive in the range of design parameter selection. But it can be seen from this data that the output performance is improved apparently, which can be also indicated from Table 7 . The output performance improvement can be explained by the percentage in Table 7, where the initial value was defined as 1.

\section{Conclusion}

This paper explored the use of intelligent optimization techniques to obtain optimum design of a multilink 


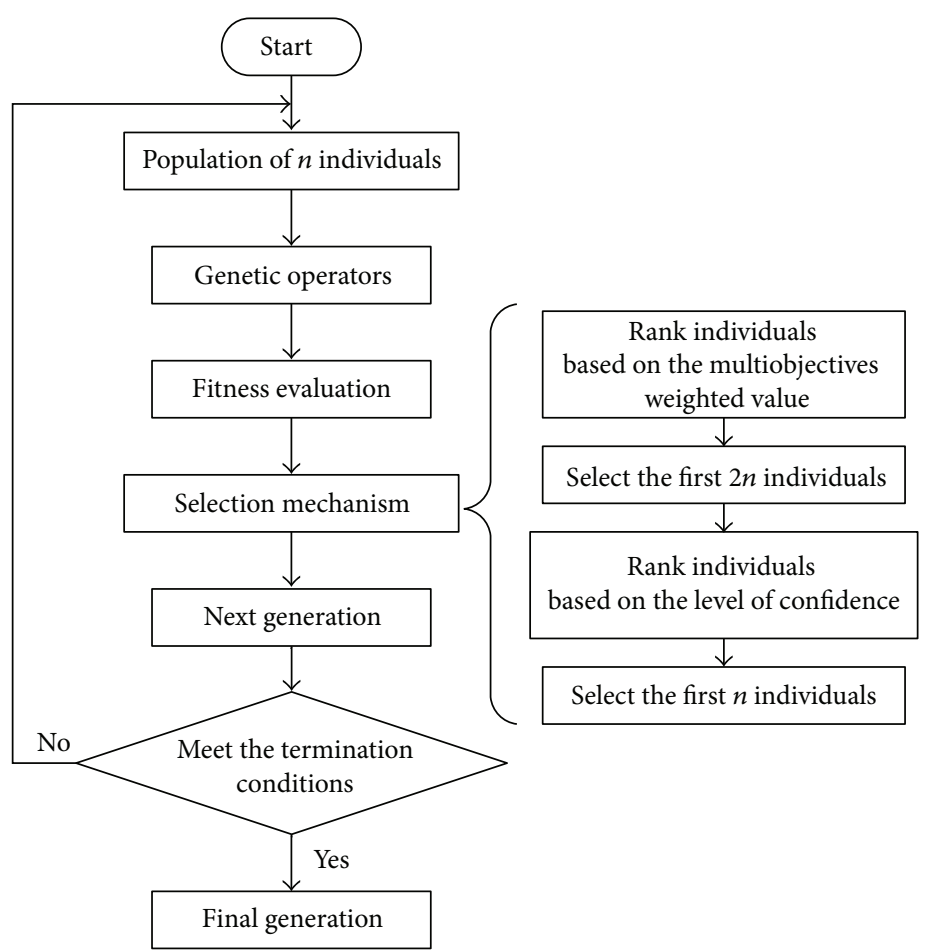

FIgURE 11: The optimization flow of the model.

TABLE 7: Comparison of output performance.

\begin{tabular}{lccc}
\hline Output parameters & Initial value & Optimized value & Performance improvement \\
\hline $\bar{v}^{\prime}$ & 1 & $0.76 \sim 0.85$ & $15 \% \sim 24 \%$ \\
$\bar{a}^{\prime}$ & 1 & $0.43 \sim 0.64$ & $36 \% \sim 57 \%$ \\
$\bar{T}^{\prime}$ & 1 & $0.53 \sim 0.57$ & $43 \% \sim 47 \%$ \\
$e_{v}^{\prime}$ & 1 & $0.54 \sim 0.58$ & $42 \% \sim 46 \%$ \\
\hline
\end{tabular}

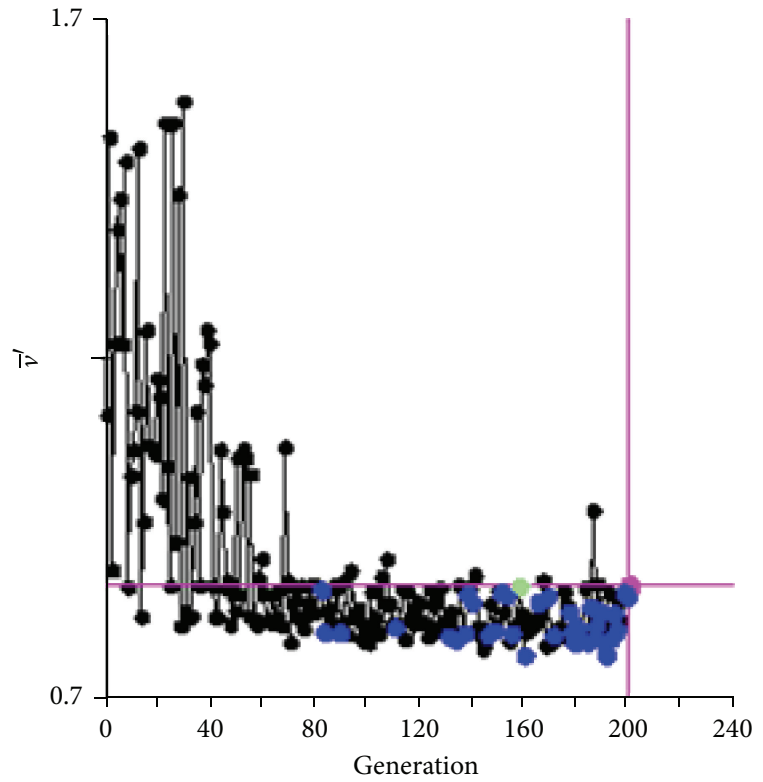

FIgURE 12: The history data of $\bar{v}^{\prime}$.

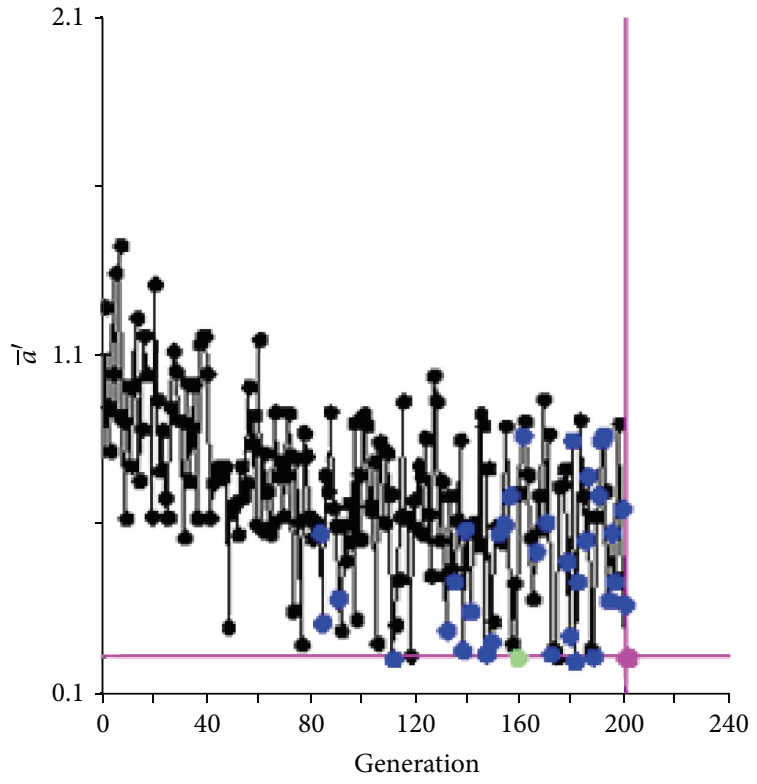

FIgURE 13: The history data of $\bar{a}^{\prime}$. 


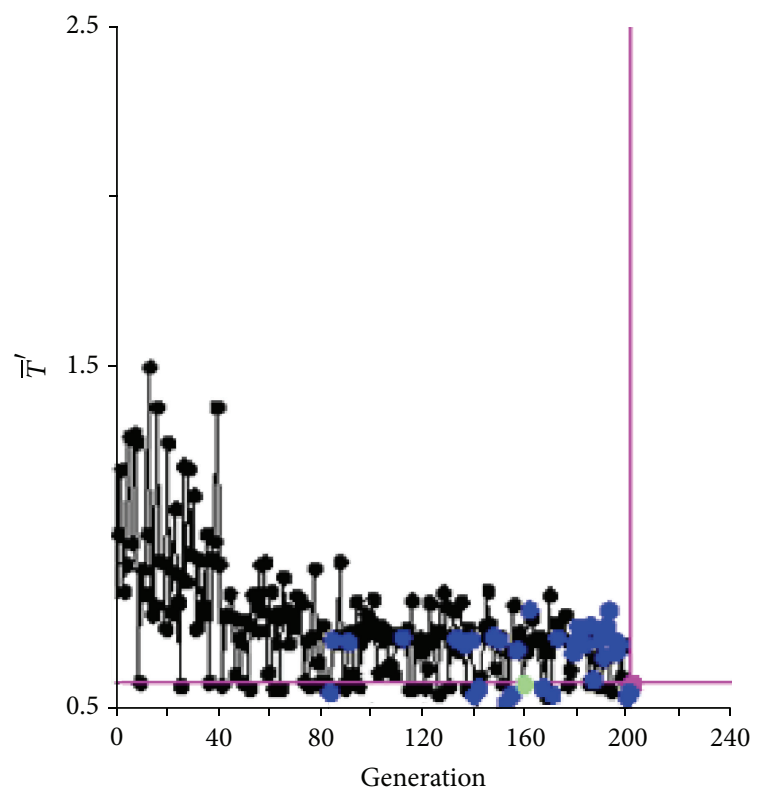

Figure 14: The history data of $\bar{T}^{\prime}$.

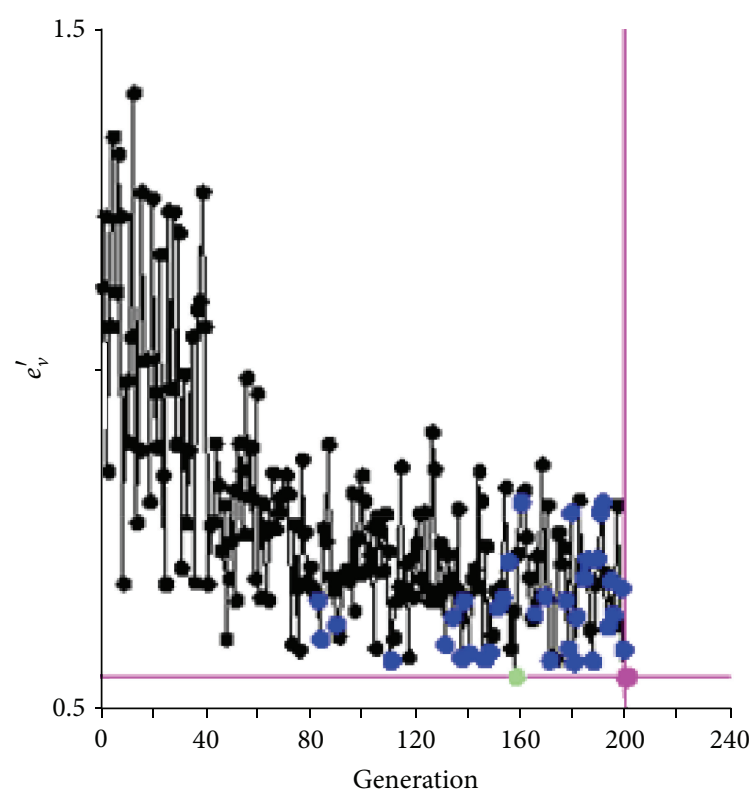

FIGURE 15: The history data of $e_{v}^{\prime}$.

transmission mechanism. In contrast with traditional evolutionary algorithms, this paper adopted a polygon model into the iterative optimization process to describe the domination relationships of the individuals on the Pareto front. In addition, robust design optimization was used in the optimization process. From the experiment results, the output performance of the multilink transmission mechanism was improved apparently. Interesting future work will include a more detailed study of the polygon model on the description of the individuals on the Pareto front.

\section{Competing Interests}

The authors declare that they have no competing interests.

\section{Acknowledgments}

This work was supported by the National Natural Science Foundation of China (no. 51505218) and the Fundamental Research Funds for the Central Universities (no. NS2015050). The supports are gratefully acknowledged.

\section{References}

[1] K. Deb, Multi-Objective Optimization Using Evolutionary Algorithms, Wiley-Interscience, New York, NY, USA, 2001.

[2] C. Coello, D. Veldhuizen, D. Van, and G. Lamont, Evolutionary Algorithms for Solving Multi-Objective Problems, Genetic Algorithms and Evolutionary Computation, Kluwer Academic, Norwell, Mass, USA, 2002.

[3] R. T. Marler and J. S. Arora, "Survey of multi-objective optimization methods for engineering," Structural \& Multidisciplinary Optimization, vol. 26, no. 6, pp. 369-395, 2004.

[4] R. Saravanan, S. Ramabalan, N. G. R. Ebenezer, and C. Dharmaraja, "Evolutionary multi criteria design optimization of robot grippers," Applied Soft Computing Journal, vol. 9, no. 1, pp. 159-172, 2009.

[5] O. Castillo, L. Trujillo, and P. Melin, "Multiple objective genetic algorithms for path-planning optimization in autonomous mobile robots," Soft Computing, vol. 11, no. 3, pp. 269-279, 2007.

[6] D. W. Gong, N. N. Qin, and X. Y. Sun, "Evolutionary optimization algorithm for multi-objective optimization problems with interval parameters," in Proceedings of 5th IEEE International Conference on Bio-Inspired Computing: Theories and Applications, pp. 411-420, Changsha, China, September 2010.

[7] M. A. Abido, "Multiobjective evolutionary algorithms for electric power dispatch problem," IEEE Transactions on Evolutionary Computation, vol. 10, no. 3, pp. 315-329, 2006.

[8] K. Deb, A. Pratap, S. Agarwal, and T. Meyarivan, "A fast and elitist multiobjective genetic algorithm: NSGA-II," IEEE Transactions on Evolutionary Computation, vol. 6, no. 2, pp. 182197, 2002.

[9] M. Emmerich, N. Beume, and B. Naujoks, "An EMO algorithm using the hypervolume measure as selection criterion," in Evolutionary Multi-Criterion Optimization: Third International Conference, EMO 2005, Guanajuato, Mexico, March 9-11, 2005. Proceedings, vol. 3410 of Lecture Notes in Computer Science, pp. 62-76, Springer, Berlin, Germany, 2005.

[10] S. Gunawan, A. Farhang-Mehr, and S. Azarm, "Multi-level multi-objective genetic algorithm using entropy to preserve diversity," in Evolutionary Multi-Criterion Optimization: Second International Conference, EMO 2003, Faro, Portugal, April 811, 2003. Proceedings, vol. 2632 of Lecture Notes in Computer Science, pp. 148-161, Springer, Berlin, Germany, 2003.

[11] R. Battiti and A. Passerini, "Brain-computer evolutionary multiobjective optimization: a genetic algorithm adapting to the decision maker," IEEE Transactions on Evolutionary Computation, vol. 14, no. 5, pp. 671-687, 2010.

[12] J. Branke, K. Deb, K. Miettinen, and R. Slowinski, Multiobjective Optimization-Interactive and Evolutionary Approaches, Springer, Heidelberg, Germany, 2008. 
[13] K. Deb, A. Sinha, P. J. Korhonen, and J. Wallenius, "An interactive evolutionary multiobjective optimization method based on progressively approximated value functions," IEEE Transactions on Evolutionary Computation, vol. 14, no. 5, pp. 723-739, 2010.

[14] H. Eskandari, C. D. Geiger, and R. Bird, "Handling uncertainty in evolutionary multiobjective optimization: SPGA," in Proceedings of the IEEE Congress on Evolutionary Computation (CEC '07), pp. 4130-4137, IEEE, Singapore, September 2007.

[15] J. W. Fowler, E. S. Gel, M. M. Köksalan, P. Korhonen, J. L. Marquis, and J. Wallenius, "Interactive evolutionary multiobjective optimization for quasi-concave preference functions," European Journal of Operational Research, vol. 206, no. 2, pp. 417-425, 2010.

[16] L. Rachmawati and D. Srinivasan, "Incorporating the notion of relative importance of objectives in evolutionary multiobjective optimization," IEEE Transactions on Evolutionary Computation, vol. 14, no. 4, pp. 530-546, 2010.

[17] L. B. Said, S. Bechikh, and K. Ghédira, "The r-dominance: a new dominance relation for interactive evolutionary multicriteria decision making," IEEE Transactions on Evolutionary Computation, vol. 14, no. 5, pp. 801-818, 2010.

[18] J. D. Schaffer, "Multiple objective optimization with vector evaluated genetic algorithms," in Proceedings of the 1st International Conference on Genetic Algorithms, pp. 93-100, Hillsdale, NJ, USA, 1985.

[19] A. Sinha, P. Korhonen, J. Wallenius, and K. Deb, "An interactive evolutionary multi-objective optimization method based on polyhedral cones," in Learning and Intelligent Optimization, C. Blum and R. Battiti, Eds., vol. 6073 of Lecture Notes in Computer Science, pp. 318-332, 2010.

[20] J. Sun, D. Gong, and X. Sun, "Solving interval multi-objective optimization problems using evolutionary algorithms with preference polyhedron," in Proceedings of the 13th Annual Conference on Genetic and Evolutionary Computation (GECCO '11), pp. 729-736, ACM, July 2011.

[21] K. Miettinen, "Survey of methods to visualize alternatives in multiple criteria decision making problems," OR Spectrum, vol. 36, no. 1, pp. 3-37, 2014.

[22] H. L. Trinkaus and T. Hanne, "KnowCube: a visual and interactive support for multicriteria decision making," Computers and Operations Research, vol. 32, no. 5, pp. 1289-1309, 2005.

[23] C. M. Fonseca and P. J. Fleming, "Genetic algorithms for multiobjective optimization: formulation discussion and generalization," in Proceedings of the 5th International Conference on Genetic Algorithms, pp. 416-423, 1993.

[24] D. E. Goldberg, Genetic Algorithms in Search, Optimization, and Machine Learning, Compact MRI for TB of the Distal Radius, 1989.

[25] N. Srinivas and K. Deb, "Multiobjective optimization using nondominated sorting in genetic algorithms," Evolutionary Computation, vol. 2, no. 3, pp. 221-248, 1994. 


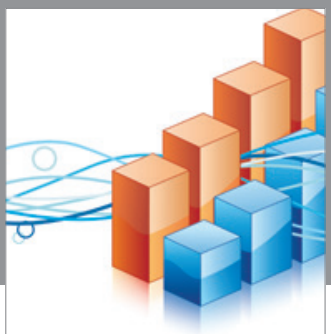

Advances in

Operations Research

vatem alat4

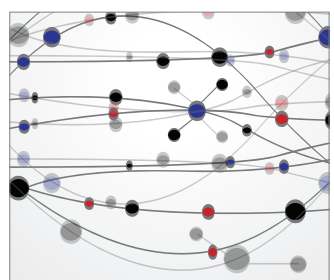

\section{The Scientific} World Journal
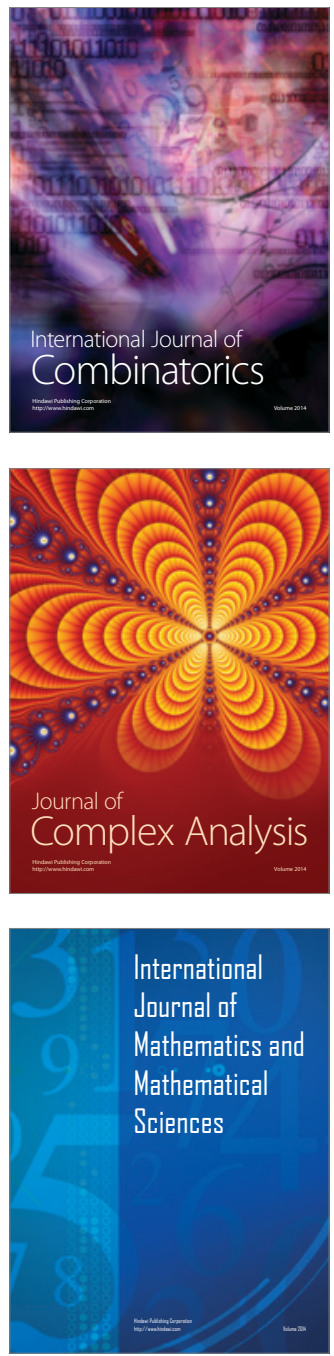
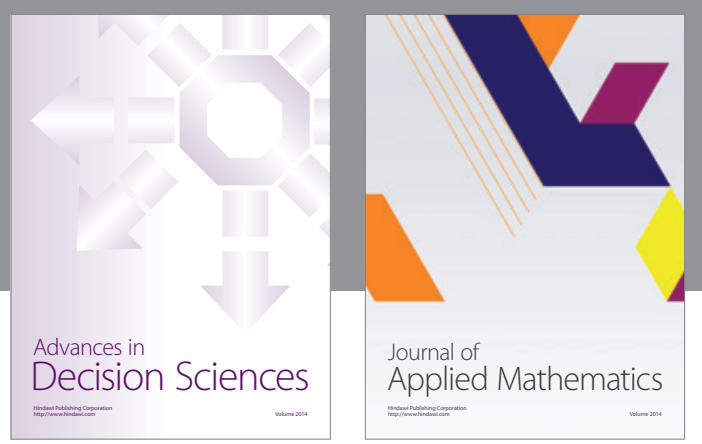

Algebra

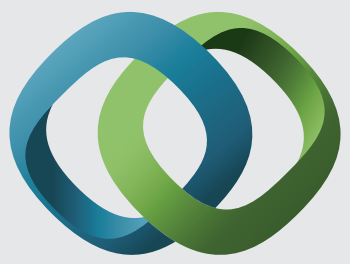

\section{Hindawi}

Submit your manuscripts at

http://www.hindawi.com
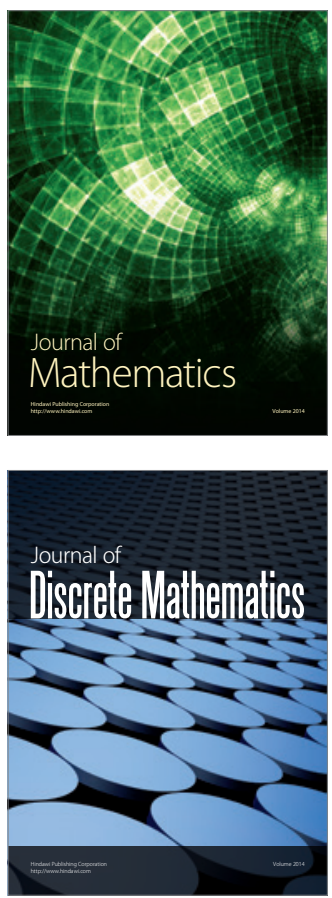

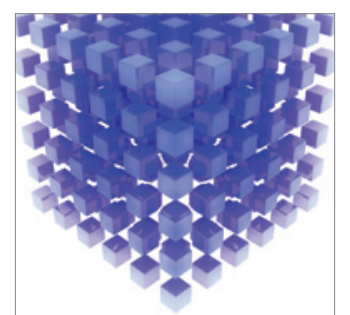

Mathematical Problems in Engineering
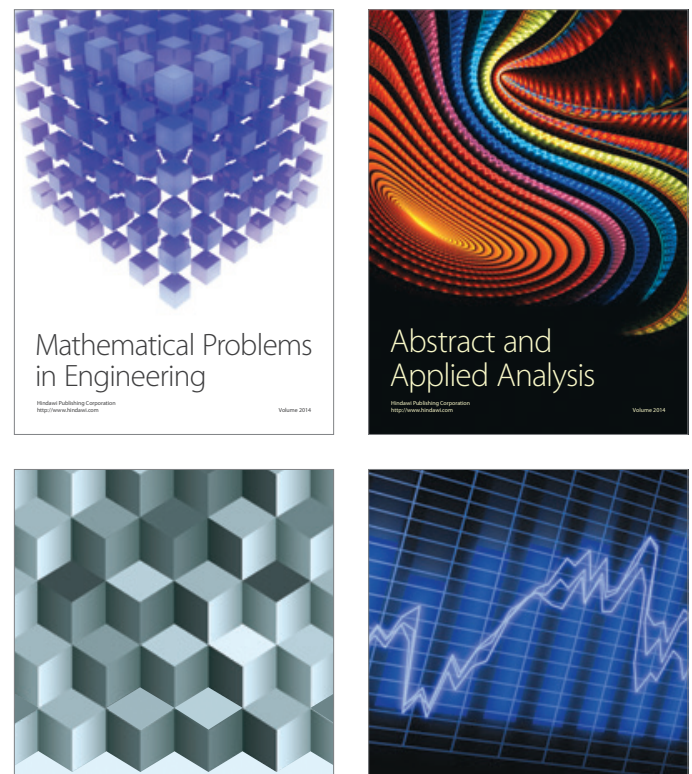

Journal of

Function Spaces

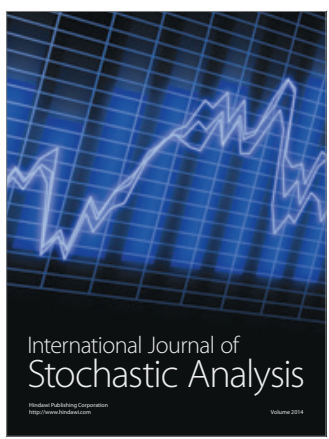

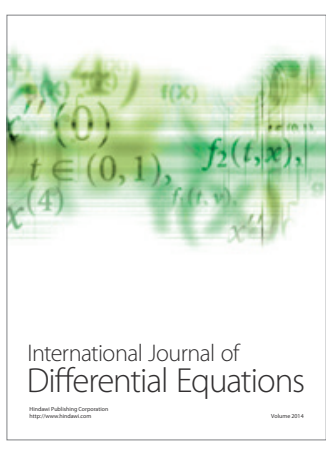
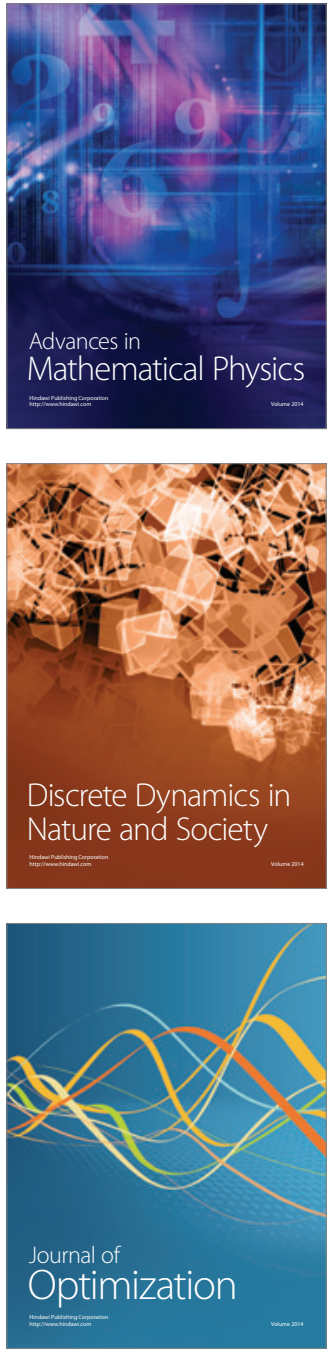